Check for updates

Cite this: RSC Adv., 2017, 7, 43502

Received 16th July 2017

Accepted 4th September 2017

DOI: 10.1039/c7ra07813g

rsc.li/rsc-advances

\section{Theoretical and experimental studies on the separation of cinnamyl acetate and cinnamaldehyde by adsorption onto a $\beta$-cyclodextrin polyurethane polymer $\dagger$}

\author{
Qinghua Liang, ${ }^{a}$ Kungang Chai, ${ }^{\star a b}$ Ke Lu, ${ }^{a}$ Zhijun Xu, ${ }^{b}$ Guoyu Li, ${ }^{a}$ Zhangfa Tong ${ }^{1}{ }^{a}$ \\ and Hongbing $\mathrm{Ji}$ (D) *ac
}

In order to isolate cinnamyl acetate (CAC) and cinnamaldehyde (CA), which coexist in cinnamon oil, a crosslinked polymer of $\beta$-cyclodextrin polyurethane (CDPU) was synthesized via a facile route and investigated as a selective adsorbent. The successful crosslinking was confirmed by FTIR, TG-DSC, XRD, SEM and $\mathrm{N}_{2}$ adsorption-desorption analysis. The adsorption mechanism was investigated through a series of instrumental analyses and theoretical calculations, indicating that the combination of noncovalent interactions (e.g., hydrogen bonding, hydrophobic interactions) accounts for the superior affinity of CAC to CDPU, compared with CA. The adsorption behaviors of CAc and CA on CDPU were performed in the static adsorption mode, in which CDPU exhibited a selective adsorption property to CAc. The adsorption kinetics and isotherms in a single component system agreed well with the pseudosecond-order kinetic model and Freundlich isotherm model, respectively. It is noteworthy that the equilibrium adsorption amount was enhanced in the presence of the co-solute, presumably resulting from the cooperative effect arising from Lewis acid-base and $\pi-\pi$ stacking interactions between the adsorbed CAC and CA molecules. Due to the stronger adsorption affinity of CAc to CDPU, adsorption enhancement of $C A C$ in the presence of $C A$ is greater than that of $C A$ in the presence of $C A C$, resulting in higher CAc/CA selectivity with increasing concentrations of the equimolar mixture of CAc and CA. Additionally, CDPU could be easily regenerated and maintained high adsorption capacities and separation efficiency even after six adsorption/desorption cycles.

\section{Introduction}

Cinnamon, mainly derived from the cinnamon tree, is commonly exploited as a natural essential oil in spices, food and medicine, cosmetic products and agricultural chemicals..$^{1-3}$ China is currently the largest exporter of cinnamon oil, and the abundant resources of cinnamon trees are mainly distributed in Southern China, especially the Guangxi Province, which accounts for over half of China's cinnamon oil exports. ${ }^{4}$ Natural cinnamon oil contains a variety of chemical components, mainly including

${ }^{a}$ School of Chemistry and Chemical Engineering, Guangxi Key Laboratory of Petrochemical Resource Processing and Process Intensification Technology, Guangxi University, Nanning 530004, PR China. E-mail: kylechild@yahoo.com; Fax: +86 771 3231382; Tel: +867713237305

${ }^{b}$ School of Light Industry and Food Engineering, Guangxi University, Nanning 530004, PR China

${ }^{c}$ Fine Chemical Industry Research Institute, School of Chemistry, Sun Yat-sen University, Guangzhou 510275, PR China. E-mail: jihb@mail.sysu.edu.cn; Fax: +86 20 84113654; +862084113658

$\dagger$ Electronic supplementary information (ESI) available: Tables (Tables S1-S3), figures (Fig. S1-S5) and text (text S1-S3) have been provided. See DOI: $10.1039 / \mathrm{c} 7 \mathrm{ra} 07813 \mathrm{~g}$ cinnamaldehyde (CA, 70-88\%), o-methoxycinnamaldehyde (4$12 \%$ ), cinnamyl acetate (CAc, $0.5-10 \%)$, and benzaldehyde (0.5$1 \%$ ), and so forth. ${ }^{5}$ Each of the major components in cinnamon oil can be widely utilized in many aspects of our society. ${ }^{6}$ For example, the main component CA is widely used in medicine, spices and chemical industry due to the characteristics of sterilization, preservation and lasting aroma. ${ }^{7-9}$ Besides, another component CAc is usually applied in the perfume, cosmetics and food industry as an important fragrance and flavor ingredient. ${ }^{\mathbf{1 0 , 1 1}}$ Therefore, it is important to obtain purified components (such as CA and CAc) from cinnamon oil. Compared to other components, it is relatively difficult to separate CA and CAc from cinnamon oil in view of their similar boiling points $\left(248\right.$ and $265^{\circ} \mathrm{C}$ for CA and CAc). In the current industry, energy-intensive, costly distillation methods (e.g., vacuum distillation, thin-film evaporation coupling distillation technology) have been employed for isolating CAc from CA. ${ }^{12}$ Taking into account the thermal sensitivity of the substrate molecules, these approaches may destroy their chemical structures, especially the unsaturated aldehyde groups of CA. Thus, it is essential to explore facile industrially applicable alternatives to isolate CA and CAc from cinnamon oil. 
Adsorption is considered as a facile and efficient approach for separation applications, due to mild conditions, easy product recovery and adsorbents regeneration. ${ }^{13,14}$ The design and construction of highly selective and inexpensive adsorbents plays a central role in adsorptive separation. ${ }^{15,16} \beta$-Cyclodextrin $(\beta$-CD) polymers, prepared by crosslinking $\beta$-CD or its derivatives with functional groups have been studied widely as adsorbents for chromatographic separation and water treatment applications. ${ }^{17-20}$ The most notable feature of $\beta$-CD polymers is the ability to selectively encapsulate a wide range of molecules due to the preservation of the hydrophobic hollow cavity that belongs to the $\beta$-CD molecule in their skeletons. ${ }^{21,22}$ Among the various investigated $\beta$-CD polymers, $\beta$-CD polyurethane (CDPU) polymers which formed by direct polymerization of $\beta$-CD's hydroxyl groups with diisocyanates, not only retain the native $\beta$-CD moiety with dramatic host-guest recognition, but also contain the carbamate crosslinking unit ensuring high mechanical strength and hydrophobicity. ${ }^{23-26}$ Moreover, due to their relatively simple manufacturing process and cost-effectiveness, CDPU polymers have been widely applied in liquid-phase adsorption systems, especially used as imprinting matrixes for selective adsorption. ${ }^{27-29}$

In this study, a CDPU polymer was easily prepared by crosslinking $\beta$-CD and toluene 2,4-diisocyanate, then used as adsorbent to separate CAc and CA. To our knowledge, no attempt has been made to separate CAc and CA by adsorption technique. The physical and chemical properties of the asprepared CDPU polymer were examined by several characteristic methods. Prior to adsorption experiments, the nature of the selective adsorption behavior was investigated based on the results of instrumental analyses and theoretical calculations. The single-component adsorption kinetics, adsorption equilibria in single- and binary-component systems, and regeneration tests were systematically investigated. This work provides a novel, convenient and efficient method for separating CAc and CA.

\section{Experimental}

\subsection{Material}

$\beta$-CD (>99\%) was purchased from Shanghai Boao Biotechnology Co., Ltd., China. Toluene 2,4-diisocyanate (TDI) was provided by Chengdu Huaxia Chemical Reagent Co. Ltd., China. Cinnamaldehyde $(\geq 99 \%)$ and cinnamyl acetate $(\geq 97 \%)$ were obtained from J\&K Chemical Co. Ltd., (Beijing, PR China). $N, N$ Dimethyl formamide (DMF, $\geq 99.5 \%$, Tianjin Guangfu Technology Development Co. Ltd) was dried with anhydrous magnesium sulfate before use. Dimethyl sulfoxide (DMSO, AR) was from Tianjin Fuyu Fine Chemical Co. Ltd., China. All water used was deionized.

\subsection{Synthesis of $\beta-C D$ polyurethane (CDPU) polymer}

$\beta$-CD (4.0 g, ca. $3.5 \mathrm{mmol})$ was dissolved in $10 \mathrm{~mL}$ of dry DMF at $60{ }^{\circ} \mathrm{C}$, and TDI $(3.1 \mathrm{~g}, \mathrm{ca} .17 .5 \mathrm{mmol})$ was added dropwise within $20 \mathrm{~min}$ under stirring (250 rpm). The resulting solution was kept under constant stirring at $60^{\circ} \mathrm{C}$ for an additional $4 \mathrm{~h}$. After accomplishing polymerization, the solid product was poured into acetone, and the resultant precipitate was filtered off and sufficiently washed with water, acetone. Then the product was dried at $60{ }^{\circ} \mathrm{C}$ for $24 \mathrm{~h}$ in vacuum and ground to fine powder. In control experiments, CDPU samples were prepared under the same conditions using different TDI dosages of $0.62 \mathrm{~g}$ (3.5 mmol), 1.86 (10.5 mmol) and $4.34 \mathrm{~g}$ (24.5 mmol), respectively. Thus, CDPU polymers with various TDI/ $\beta$-CD molar ratio from $1 / 1$ to $7 / 1$ were fabricated.

\subsection{Characterization}

FTIR spectra were recorded on a Bruker TENSOR II FTIR spectrophotometer using samples diluted in $\mathrm{KBr}$ pressed pellets over the frequency range of $4000-400 \mathrm{~cm}^{-1}$. Power X-ray diffraction (XRD) patterns were collected on a X'Pert PRO diffractometer (PANalytiCA B.V., Holland), using $\mathrm{Cu} \mathrm{K} \alpha$ radiation $(\lambda=1.54060 \AA)$ at $40 \mathrm{kV}, 30 \mathrm{~mA}$. The specific surface area and the pore distribution were measured at $-196{ }^{\circ} \mathrm{C}$ using an ASAP 2020 sorptometer (Micromeritics, USA). Scanning electron microscopic (SEM) imaging was conducted using a Hitachi scanning electron microscope at $10 \mathrm{kV}$ (Hitachi S-3400, Japan). TG-DSC curves were performed on a NETZSCH instrument (NETZSCH STA 449F5, Germany). The samples were heated up to $850{ }^{\circ} \mathrm{C}$ under nitrogen flow, at a heating rate of $10{ }^{\circ} \mathrm{C} \mathrm{min}^{-1}$. Solid-state 13C CP/MAS NMR spectra were recorded on a Varian Inova-500 NB spectrometer at $125.72 \mathrm{MHz}$, with a contact time of $2 \mathrm{~ms}$, a spinning rate of $7 \mathrm{kHz}$, and a recycling time of $12 \mathrm{~s}$ at ambient temperature. X-ray photoelectron spectroscopy (XPS) analysis was carried out on a Vacuum Generator ESCAAB 250 spectrometer with $\mathrm{Al}$ mono $\mathrm{K} \alpha \mathrm{X}$-ray source to investigate the $\mathrm{C}, \mathrm{N}$, and $\mathrm{O}$ atoms present on surface of CDPU, with a reduced power of $150 \mathrm{~W}$ and the pressure in the analysis chamber at less than $10^{-8}$ Torr.

\subsection{Quantification in solution}

The concentrations of CAc and CA in aqueous solution were quantified using liquid-phase extraction followed by gas chromatography (GC). Typically, a fixed volume of aqueous solution was withdrawn and extracted with the same volume of ethyl acetate containing the internal standard naphthalene. The concentrations of CAc and CA in extractant were analyzed by GC (Shimadzu GC smart 2018) equipped with flame ionization detector using a Wonda Cap 1 column $(30 \mathrm{~m} \times 0.25 \mathrm{~mm} \times$ $0.25 \mu \mathrm{m})$. The chromatographic conditions were as follows: nitrogen was used as carrier gas; detector temperature and injector temperature, $260{ }^{\circ} \mathrm{C}$; column temperature, $170{ }^{\circ} \mathrm{C}$. Under these conditions, the retention times were $4.01 \mathrm{~min}$ for naphthalene, $4.34 \mathrm{~min}$ for $\mathrm{CA}$, and $6.00 \mathrm{~min}$ for CAc, respectively. All measurements were performed in triplicate and the means were used.

\subsection{Adsorption experiments}

The single-component solution of CAc or CA was prepared by dissolving certain amount of CAc or CA into $40 \%$ DMSO aqueous solution (v/v). For preparing equimolar binarycomponent solution of CAc and CA, different amounts of the 
equimolar mixture of CAc and CA were dissolved into the same solvent. All the batch adsorption experiments were performed in a water bath with shaker at $150 \mathrm{rpm}$. Each experiment was conducted in triplicate and the mean values and standard deviations are reported.

2.5.1 Single-component adsorption kinetic experiments. The adsorption kinetic experiments were performed as follows: $2 \mathrm{~g}$ of CDPU with $200 \mathrm{~mL}$ of CAc or CA solution $\left(25 \mathrm{mmol} \mathrm{L}^{-1}\right)$ were placed in a $500 \mathrm{~mL}$ conical flask, then shaken at $25{ }^{\circ} \mathrm{C}$. At various time intervals, aliquots $(1 \mathrm{~mL})$ were withdrawn from the flask and immediately centrifuged at $4000 \mathrm{rpm}$ for $5 \mathrm{~min}$. The content of CAc or CA in the supernatant was examined as described above. The adsorbed amounts of CAc or CA $\left(q_{t}, \mathrm{mmol}^{-1}\right)$ at different time were calculated by the following equation:

$$
q_{t}=\left(C_{0}-C_{\mathrm{t}}\right) V / m
$$

where $C_{0}, C_{\mathrm{t}}, V$, and $m$ are the initial concentration of guest $\left(\mathrm{mmol} \mathrm{L}^{-1}\right)$, the concentration of guest $\left(\mathrm{mmol} \mathrm{L}^{-1}\right)$ at time $t$, the initial volume of solution (L), and the weight of adsorbent $(\mathrm{g})$, respectively.

2.5.2 Single-component adsorption isotherm experiments. For single-component adsorption isotherm experiments, the initial concentration of CAc or CA in the single-solute solution varied from 15 to $35 \mathrm{mmol} \mathrm{L}{ }^{-1} \cdot 0.2 \mathrm{~g}$ of CDPU was added into $10 \mathrm{~mL}$ of the single-component solution and shaken for $4 \mathrm{~h}$ at 25,35 and $45{ }^{\circ} \mathrm{C}$, respectively. After attaining equilibrium, the equilibrium concentration of CA or CAc in the supernatant was analyzed. The equilibrium adsorption capacity $q_{\mathrm{e}}\left(\mathrm{mmol} \mathrm{g}^{-1}\right)$ was calculated according to eqn (2).

$$
q_{\mathrm{e}}=\left(C_{0}-C_{\mathrm{e}}\right) V / m
$$

where $C_{0}$ and $C_{\mathrm{e}}$ are the initial and equilibrium concentrations of guests ( $\mathrm{mmol} \mathrm{L}^{-1}$ ), $V$ is the volume of solution (L), and $m$ is the weight of adsorbent $(\mathrm{g})$.

2.5.3 Binary-component adsorption isotherm experiments. In the binary-component equilibrium adsorption experiments, CDPU (0.2 g) was added into $10 \mathrm{~mL}$ of equimolar CAc-CA solutions with varying concentrations in the range of 30-70 $\mathrm{mmol} \mathrm{L}^{-1}$, being shaken at $25{ }^{\circ} \mathrm{C}$ for $4 \mathrm{~h}$. The suspension solutions were then filtered and analyzed. The equilibrium adsorption capacity $q_{\mathrm{e}}\left(\mathrm{mmol} \mathrm{g}^{-1}\right)$ was calculated by eqn (2), and selectivity ( $\alpha$, dimensionless) was calculated on the basis of eqn (3): ${ }^{30}$

$$
\alpha=\left(q_{\mathrm{e}, \mathrm{CAc}} \times C_{\mathrm{e}, \mathrm{CA}}\right) /\left(q_{\mathrm{e}, \mathrm{CA}} \times C_{\mathrm{e}, \mathrm{CAc}}\right)
$$

where $q_{\mathrm{e}, \mathrm{CAc}}$ and $q_{\mathrm{e}, \mathrm{CA}}$ are the equilibrium sorption capacities of CAc and CA (mmol $\left.\mathrm{g}^{-1}\right), C_{\mathrm{e}, \mathrm{CAc}}$ and $C_{\mathrm{e}, \mathrm{CA}}$ are the equilibrium concentrations of CAc and CA $\left(\mathrm{mmol} \mathrm{L}^{-1}\right)$.

2.5.4 Desorption and recycling experiments. In the adsorption process, $0.2 \mathrm{~g}$ of CDPU was added in $10 \mathrm{~mL}$ of equimolar CAc-CA solution $\left(70 \mathrm{mmol} \mathrm{L}^{-1}\right)$, being shaken at $25{ }^{\circ} \mathrm{C}$ and $150 \mathrm{rpm}$ for $4 \mathrm{~h}$. After adsorption, the sorbent was separated from supernatant by filtration. To strip the solutes from CDPU, the spent sorbent was immersed in $20 \mathrm{~mL}$ of ethyl acetate, and the suspension was vigorously shaken at $25^{\circ} \mathrm{C}$ for $3 \mathrm{~h}$. Then the regenerated CDPU was retrieved by filtration, followed by drying at $60{ }^{\circ} \mathrm{C}$ for $24 \mathrm{~h}$ in vacuum. To evaluate the reversibility of CDPU, six consecutive cycles of adsorption experiments were experimentally demonstrated.

2.5.5 Adsorption experiments using different adsorbents. For comparison, four adsorbents including the commercial bentonite and activated carbon (AC), $\beta$-CD crosslinked hexamethylene diisocyanate polymer (CHP) and $\beta$-CD crosslinked epichlorohydrin polymer (CEP) were used. And the specific information for these adsorbents were listed in the ESI. $\uparrow$ The adsorption experiments were performed with $10 \mathrm{~mL}$ of equimolar CAc-CA solutions $\left(70 \mathrm{mmol} \mathrm{L}^{-1}\right)$ in $50 \mathrm{~mL}$ flasks. Exactly $0.2 \mathrm{~g}$ of different adsorbents was added to each flask, and the solution was shaken at $25{ }^{\circ} \mathrm{C}$ for $4 \mathrm{~h}$. Then the suspension solutions were treated as the procedure as described in Section 2.5.3.

\subsection{Computational methods}

Before adsorption experiments, we carried out density functional theory (DFT) calculations to investigate the theoretical feasibility of using CDPU as adsorbent to separate CAc and CA. Theoretical calculations were carried out with the program package DMol3 in Materials Studio (version 8.0 from Accelrys Software Inc., United States). The calculation was performed assuming that one $\beta$-CD molecular connected with two crosslink units to simulate the basic unit of CDPU structure. The geometry optimization of Dmol3 calculations were constructed based on local density approximation (LDA) in the PerdenWang (PWC), and all-electron Kohn-Sham wave functions were expanded in a double numerical basis set including the polarization function (DND). ${ }^{9}$ All simulations were performed with a max step size of $0.3 \AA$, the max interaction was 1000 times, the SCF tolerance was 0.00001 and the max SCF cycles was 50 . Besides, the orbital cutoff is $3.3 \AA$, and the other parameters were set to default for geometry optimization. Before the DFT calculation, the structures of CA molecule, CAc molecule and CDPU unit were fully optimized, and the optimum geometries are shown in Fig. S1. $\dagger$ In the DFT calculation, the adsorption behaviors in the horizontal binding of the guests onto crosslink unit and encapsulation of guests into $\beta$-CD cavity were investigated, respectively. In terms of inclusion into $\beta$-CD cavity, the oxygen-containing functional groups of guests entering from $\beta$-CD's narrow side (the primary hydroxyl groups side) was named 'head up' and entering from its wide side (the secondary hydroxyl groups side) was named 'head down'. To compare the stabilities of the studied complex structures, the binding energy, $E_{\mathrm{b}}$, of the minimum energy structures were calculated using the following formula:

$$
E_{\mathrm{b}}=E_{\mathrm{com}}-\left(E_{\mathrm{CDPU}}+E_{\mathrm{ads}}\right)
$$

where $E_{\mathrm{CDPU}}, E_{\mathrm{ads}}$ and $E_{\mathrm{com}}$ in $\mathrm{kJ} \mathrm{mol}^{-1}$ stand for the energies of CDPU unit, guest and complexes, respectively.

To verify the reliability of the calculating results by MS, the binding configurations above were optimized with PM3 followed by ONIOM2 calculation in Gaussian 09 computer program. The substrate molecules were fully optimized using 
a DFT methodology employing the B3LYP function with 6$31 \mathrm{G}(\mathrm{d})$ basis set, and the optimization of the CDPU unit was performed with a semiempirical method PM3. In order to keep consistent with Dmol3 in Materials Studio, the simulations were performed with a max step size of $0.3 \AA$, the max interaction was 1000 times, and the other parameters were default values. Finally, the calculated results were substituted into eqn (4) to obtain the binding energy.

\section{Results and discussion}

\subsection{Characterization of pristine CDPU}

The structural characterization of the as-prepared polymer was performed using various techniques by comparison with $\beta$-CD as a reference. The FTIR spectrum of $\beta$-CD displays the bands at 3372,2926 , and $1031 \mathrm{~cm}^{-1}$ for the $\mathrm{O}-\mathrm{H}, \mathrm{C}-\mathrm{H}$ and $\mathrm{C}-\mathrm{O}$ stretching modes as well as the $\mathrm{O}-\mathrm{H}$ bending vibration at $1644 \mathrm{~cm}^{-1}$ (Fig. 1a). For CDPU, the characteristic peaks of $\beta$-CD are retained in its spectrum, and there are some new peaks at 3295, $1650,1603,1240$ and $1544 \mathrm{~cm}^{-1}$, which are related to the stretching vibrations of $\mathrm{N}-\mathrm{H}, \mathrm{C}=\mathrm{O}$, phenyl rings and $\mathrm{C}-\mathrm{O}-\mathrm{C}$, bending vibrations of amide groups, respectively. ${ }^{31}$ TG-DSC curves (Fig. 1b) of $\beta$-CD and CDPU show a slight weight loss in the temperature range of $25-150{ }^{\circ} \mathrm{C}$ which corresponds to the evaporation of adsorbed water, followed by a plateau region until $290{ }^{\circ} \mathrm{C}$, where a large weight loss starts, indicating decomposition of the materials. During the second weight loss stage, an endothermic peak in DSC curve is also observed at $297.4{ }^{\circ} \mathrm{C}$ for $\beta$-CD and $318.7{ }^{\circ} \mathrm{C}$ for $\mathrm{CDPU}$, respectively. Compared to $\beta$-CD, CDPU exhibits higher decomposition (a)

(c)
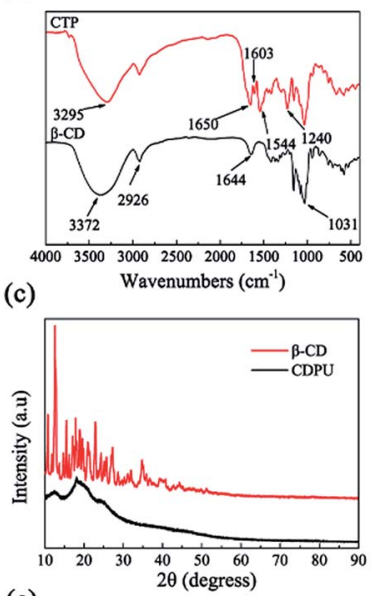

(e)

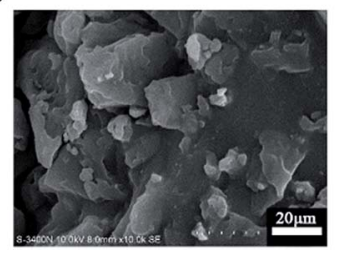

(b)

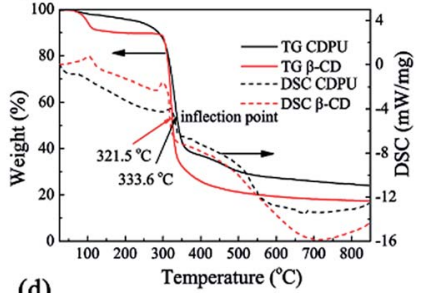

(d)

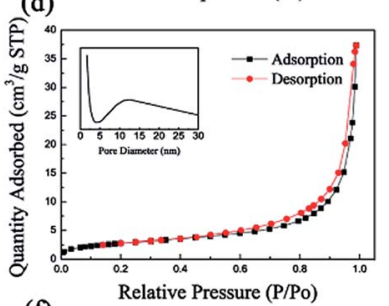

(f)

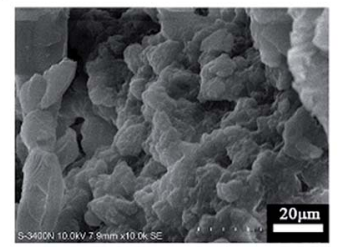

Fig. 1 FTIR spectra (a), TG-DSC curves (b) and XRD patterns (c) of $\beta$ $C D$ and CDPU, (d) nitrogen adsorption-desorption isotherms of CDPU, SEM of (e) $\beta$-CD, (f) CDPU. temperature, which may be related to the more thermo-stable of its network structure. The wide-angle XRD pattern of $\beta$-CD (Fig. 1c) presents many sharp diffraction peaks from $10.0^{\circ}$ to $28.0^{\circ}$ (The main $2 \theta$ values are, $10.6^{\circ}, 12.4^{\circ}, 15.4^{\circ}, 17.1^{\circ}, 18.9^{\circ}$, $19.5^{\circ}, 22.7^{\circ}$ etc. .) ${ }^{23}$ Compared with $\beta$-CD, only two amorphous peaks with the equivalent Bragg angles at $2 \theta=12.4^{\circ}, 18.9^{\circ}$ were recorded in the XRD pattern of CDPU, indicating the reduction in crystallinity after crosslinking reaction. The lack of crystalline structure in CDPU may be attributed to the loss of regularity throughout the polymeric chains due to the introduction of bulky TDI molecules. ${ }^{32}$ The porosity of CDPU was confirmed by reversible gas sorption experiments. A nitrogen adsorptiondesorption isotherm at $-196{ }^{\circ} \mathrm{C}$ (Fig. 1d) exhibits slow increase at low $\mathrm{N}_{2}$ pressures, a typical type III behavior of mesoporous, followed by quick saturation at high pressures. The BET surface area and pore volume of CDPU calculated from the isotherm were $10.19 \mathrm{~m}^{2} \mathrm{~g}^{-1}$ and $0.0574 \mathrm{~cm}^{3} \mathrm{~g}^{-1}$, which are larger than that of the original $\beta$-CD (data were collected in Table S1 $\dagger$ ). The pore size distribution (Fig. 1d insert) obtained by BJH method showed a wide range of pore distribution from 3 to $30 \mathrm{~nm}$. The SEM was used to observe microscopic morphological structures of $\beta$-CD and CDPU (Fig. 1e and f). Compared with the surface features of $\beta-\mathrm{CD}$, the granules of CDPU exhibit a spongy and rough surface. On the basis of the above-mentioned results, it is obvious that the polymerization between $\beta$-CD and TDI has occurred and the CDPU polymer was successfully prepared.

\subsection{Characterization of CDPU after adsorption}

3.2.1 ${ }^{13} \mathrm{C} \mathrm{CP/MAS} \mathrm{NMR} \mathrm{analysis.} \mathrm{In} \mathrm{this} \mathrm{work,} \mathrm{the} \mathrm{analysis}$ of the interaction between CDPU and CAc or CA is crucial. The ${ }^{13} \mathrm{C}$ CP/MAS NMR spectroscopy was applied to confirm the successful adsorption of CAc and CA onto CDPU. In Fig. 2a, the

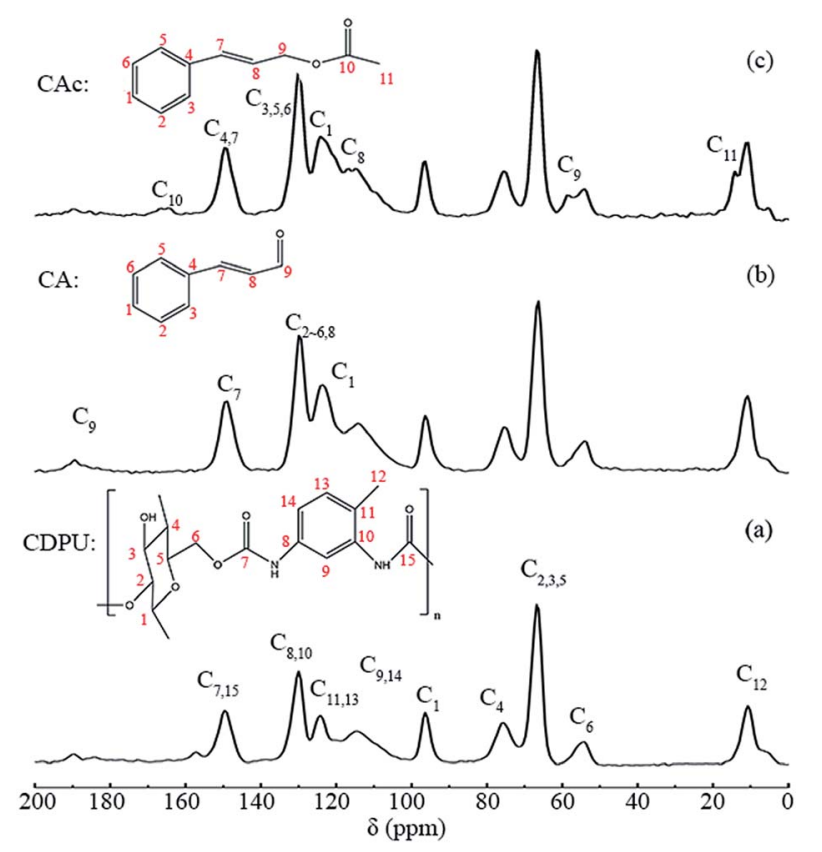

Fig. $2{ }^{13} \mathrm{C}$ CP/MAS NMR spectra of CDPU before (a), and after adsorption of CA (b) or CAc (c). 
spectrum of CDPU exhibits the resonance signals for all carbon atoms present in the structure of $\beta-\mathrm{CD}$, at $\delta=54,66,76$ and $96 \mathrm{ppm}$, which is similar to the previous report. ${ }^{33}$ Meanwhile, the spectrum also exhibits resonances associated with aromatic carbamate unit in CDPU at 11, 20, 30, 115, 124, 130 and 150 ppm. After CA or CAc being adsorbed onto CDPU (Fig. 2b and $\mathrm{c}$ ), the intensities of phenyl resonances at 124, 130 and $150 \mathrm{ppm}$ are significantly enhanced. In addition, after adsorption of CAc on CDPU, some new signals corresponding to $\mathrm{C}_{11}$, $\mathrm{C}_{9}, \mathrm{C}_{8}$ of CAc appeared at 14, 59 and 117 ppm, respectively. According to all the above mentioned, we can confirm that guest molecules have been adsorbed onto CDPU.

3.2.2 XPS analysis. The interaction between substrate molecules and CDPU was further verified by XPS. The highresolution $\mathrm{C} 1 \mathrm{~s}$ and $\mathrm{O} 1 \mathrm{~s}$ spectra of CDPU before and after adsorption of CAc or CA are presented in Fig. 3, and the corresponding XPS spectra of survey scan and N1s spectra are provided in the ESI (Fig. S2 $\dagger$ ), besides, the relative contents of deconvoluted components are listed in Table S2. $\dagger$ The C1s spectrum of CDPU can be deconvoluted into four peaks at 284.8, 285.6, 286.5 and $288.6 \mathrm{eV}$, arising from $\mathrm{C} 1\left(\mathrm{C}^{*}-\mathrm{C} / \mathrm{C}^{*}-\mathrm{H}\right), \mathrm{C} 2\left(\mathrm{C}^{*}\right.$ $\mathrm{NH}-\mathrm{C}=\mathrm{O}), \quad \mathrm{C} 3 \quad\left(\mathrm{C}^{*}-\mathrm{OH} / \mathrm{C}^{*}-\mathrm{O}-\mathrm{C}\right)$, and $\mathrm{C} 4 \quad\left(\mathrm{~N}-\mathrm{C}^{*}(\mathrm{O})=\mathrm{O}\right)$, respectively. ${ }^{34}$ After adsorption of $\mathrm{CAc}$ or $\mathrm{CA}$, we can clearly observe a notable increase of the relative content of $\mathrm{C} 1$ and a significant decrease of the relative content of C2. For the O1s spectrum of CDPU, there exist two peaks at $531.1 \mathrm{eV}$ and $533.3 \mathrm{eV}$, which are denoted as $\mathrm{O} 1\left(\mathrm{C}-\mathrm{NH}-\mathrm{C}=\mathrm{O}^{*}\right)$ and $\mathrm{O} 2(\mathrm{C}-$ $\left.\mathrm{O} * \mathrm{H} / \mathrm{C}-\mathrm{O}^{*}-\mathrm{C}\right)$, respectively. After adsorption of CAc, the relative content of $\mathrm{O} 1$ increased apparently due to the abundance of ester group in the adsorbed molecules of CAc. On the other hand, in the O1s spectrum of CDPU/CA, a new peak appeared at $532.3 \mathrm{eV}$ (denoted as O3) due to the introduction of aldehyde
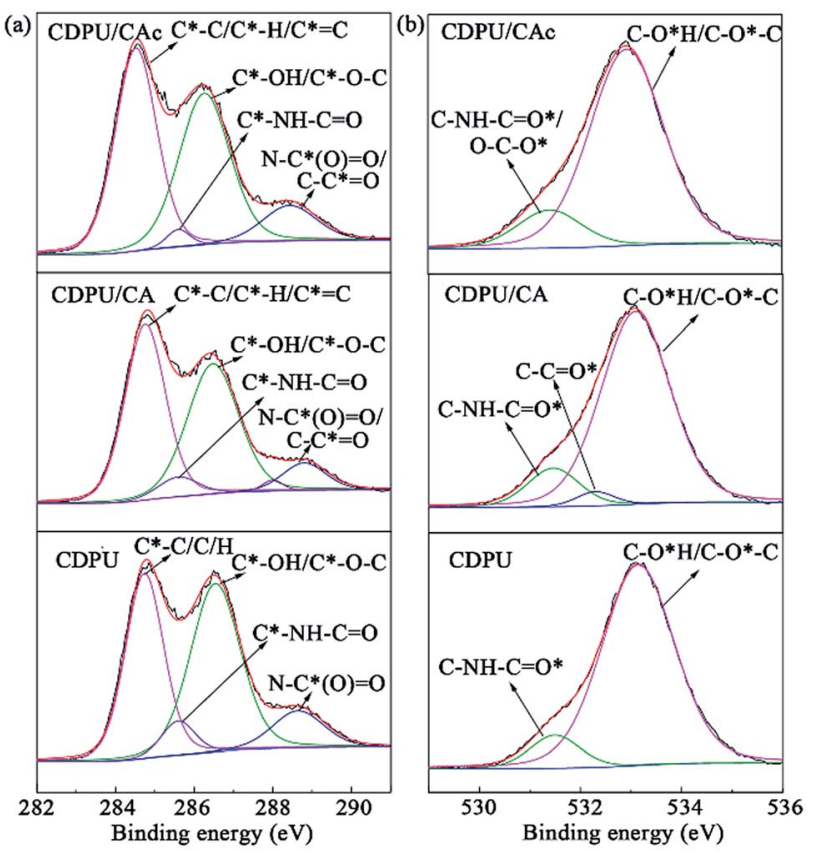

Fig. 3 XPS C1s (a) and O1s (b) of CDPU before and after adsorption of CA or CAC.

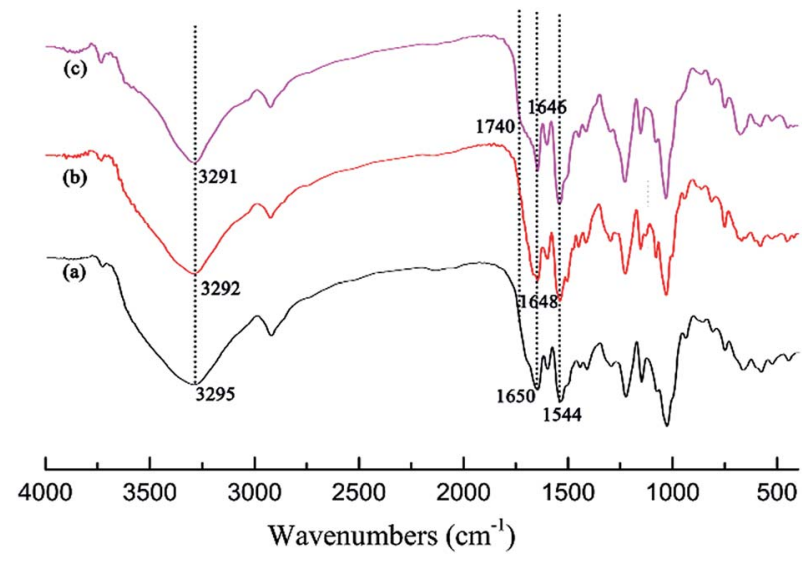

Fig. 4 FTIR spectra: (a) CDPU, (b) CA adsorbed onto CDPU, (c) CAC adsorbed onto CDPU.

group in CA. In contrast to the C1s and O1s spectra, in the N1s spectrum of CDPU (Fig. S2 $\dagger$ ), the single deconvoluted peak (C$\mathrm{N}^{*} \mathrm{H}-\mathrm{C}=\mathrm{O}$ ) at $c a .400 .12 \mathrm{eV}$ remains essentially unchanged during and after adsorption, attributed to the absence of nitrogen contained group in the substrate molecules. In addition, the binding energies of all peaks in the spectrum of CDPU remain virtually unchanged after adsorption, indicating that main forces for adsorption are non-covalent interactions.

3.2.3 FTIR analysis. To further identify possible interactions occurring between CDPU and guest molecules, the FTIR spectra of CDPU before and after adsorption were recorded (Fig. 4). In the spectrum of CDPU/CAc, there existed a new peak at $1740 \mathrm{~cm}^{-1}$ as a shoulder of $\mathrm{C}=\mathrm{O}$ stretching vibration at $c a$. $1650 \mathrm{~cm}^{-1}$, corresponding to the stretching vibration of ester groups in CAc molecules. Moreover, after adsorption of CAc or $\mathrm{CA}$, the stretching vibrations of $\mathrm{N}-\mathrm{H}\left(3295 \mathrm{~cm}^{-1}\right)$ and $\mathrm{C}=\mathrm{O}$ $\left(1650 \mathrm{~cm}^{-1}\right)$ both slightly shift to lower frequency. Such redshift in the characteristic bands of polar functional groups is a usual indication for the existence of intermolecular hydrogen bond in the solid state. ${ }^{35}$ Thus, we can infer that intermolecular hydrogen bonds have been formed during the adsorption process. Furthermore, larger red-shift of $\nu(\mathrm{N}-\mathrm{H})$ and $\nu(\mathrm{C}=\mathrm{O})$ can be observed in the spectrum of CDPU/CAc than that of $\mathrm{CDPU} / \mathrm{CA}$, indicating that the former forms stronger intermolecular hydrogen bonding interaction than the latter.

\subsection{Analysis of theoretical calculations}

To investigate the nature of the interaction between CAc or CA and the adsorption sites of CDPU, we turned to quantum chemical calculation. The optimized adsorption configurations between guest molecules and adsorption sites as well as the calculated binding energies are summarized in Fig. 5. The results show that the binding energies of CAc onto adsorption sites are always higher than that of CA, which accounts qualitatively for the superior affinity of CAc to CDPU over CA. Additionally, compared to the adsorption of guests onto the crosslink unit, the encapsulation of guests into the $\beta$-CD cavities exhibits a higher binding energy, indicating that the guest 


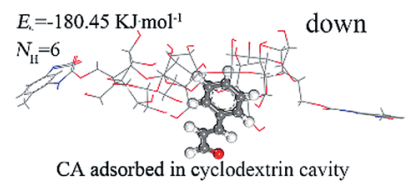

$E_{\mathrm{b}}=-260.87 \mathrm{KJ} \cdot \mathrm{mol}^{-1}$

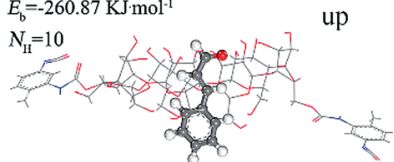

CA adsorbed in cyclodextrin cavity

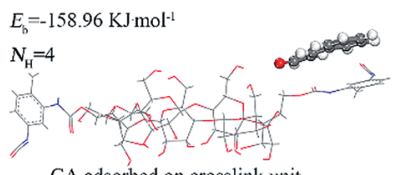

$\mathrm{CA}$ adsorbed on crosslink unit

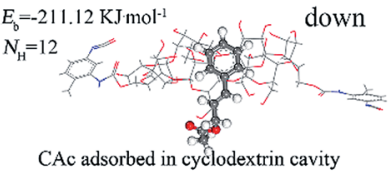

CAc adsorbed in cyclodextrin cavity $E_{\mathrm{b}}=-293.65 \mathrm{KJ} \mathrm{mol}^{-1}$ $N_{\mathrm{H}}=13$

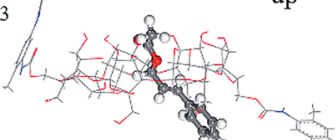

CAc adsorbed in cyclodextrin cavity

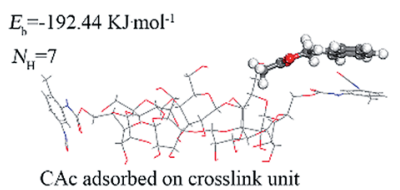

CAc adsorbed on crosslink unit

Fig. 5 Optimized geometries and binding energy, numbers of intermolecular hydrogen bonds between CDPU and guests obtained by Dmol3.

molecules are more likely to be included in the $\beta$-CD cavities. As for the complexation of $\beta$-CD and guests, the binding energy in the head up mode is higher than that of head down mode, which may be assigned to the fact that more hydrogen bonds could be formed between the oxygen-containing functional groups of the guest molecules and the secondary hydroxyl groups on the wide side of $\beta$-CD cavities.

The calculated binding energies $\left(E_{\mathrm{b}}\right)$ by Gaussian calculations are shown in Fig. S3. $\dagger$ The results show that the binding energies of CAc onto adsorption sites are always higher than that of CA, which is consistent with the results of Dmol3 and accounts qualitatively for the superior affinity of CAc to CDPU over CA. The binding energies by Gaussian calculations are remarkably higher than the results obtained from the LDA/ PWC/DND calculations. This may be attributed to the hybrid DFT methods, such as B3LYP, is more complex and fine compared to the pure DFT, such as LDA. Thus, the configuration obtained by hybrid DFT methods is more stable than of pure DFT. In addition, by comparing the binding energies by Gaussian calculations and MS calculated, the difference is in the most likely adsorption sites, for Gaussian is head down, but for MS is head up. Therefore, based on the results of theoretical calculation, we can make a conclusion that CDPU has a stronger affinity for CAc than CA.

Upon comparing the binding energies based on Dmol3 between CDPU units and guest molecules, the intermolecular hydrogen bonding interactions, existing in the optimized adsorption configurations, were also analyzed. The detailed results in terms of the detailed types, numbers, lengths and angles of hydrogen bond are listed in Table $\mathrm{S} 3, \dagger$ and the number of hydrogen bonds $\left(N_{\mathrm{H}}\right)$ between the guests and the adsorption sites in the optimized adsorption geometries are listed in Fig. 5. It is clear that hydrogen bonds between CAc and adsorption sites of CDPU are more abundant than CA-CDPU adsorption system, which shows that hydrogen bonding interaction plays an important role in the selective adsorption of CAc over CA.

\subsection{Adsorption properties of CAc and CA onto CDPU}

The adsorption performance of CDPU toward CAc and CA has been investigated, including sorption kinetics, isotherms, thermodynamics, selective sorption in binary-component systems, and regeneration of CDPU.

Firstly, due to the low solubility of CAc and CA in aqueous solution, it is desirable to introduce organic solvent to enhance their solubility. In this study, we therefore chose a range of organic solvents that are low-toxic, miscible with water, inexpensive and commercially available. In addition, the degree of crosslinking of polymer can affect the adsorption performance of CDPU substantially. Before the single-component adsorption experiments, we investigated the effects of the co-solvent composition and the degree of crosslinking on the performance of CDPU in the adsorptive separation of CAc and CA. The detailed conditions and results are collected in the ESI. $\uparrow$

Fig. S $3 \uparrow$ shows not only the strong preference of CDPU for CAc over CA, but shows also an increase of the maximum uptake levels as the co-solvent becomes less hydrophobic in nature. A high affinity is desirable in purification applications to efficiently adsorb guests. ${ }^{36}$ Compared to other co-solvents, CDPU shows the best adsorptive separation performance on CAc and CA in the $40 \%$ DMSO aqueous solution (v/v). Therefore, we chose this mixed solvent system as an excellent medium in the following adsorption experiments. As for the effect of degree of crosslinking, the equilibrium adsorption of CAc and CA onto CDPU with different degrees of crosslinking (in terms of different molar ratios of TDI to $\beta-\mathrm{CD}$ ) is shown in Fig. S5. $\dagger$ The adsorption amounts and selectivity of CAc over CA onto CDPU increase gradually with increasing molar ratio of TDI $\beta$-CD from $1 / 1$ to $5 / 1$. The enhanced adsorption of guests onto CDPU is probably because of the introduction of more binding site into the network structure of CDPU with the higher molar ratio of TDI/ $\beta$-CD. However, the further increasing of TDI/ $\beta$-CD molar ratio from $5 / 1$ to $7 / 1$ results virtually no change of the adsorption amounts of guests. Moreover, the high molar ratio of TDI/ $\beta$ $\mathrm{CD}$ at $7 / 1$ contributes to high rigidity of the polymer, so the polymer is difficult to be ground into beads. Thus, the optimum molar ratio of TDI/ $\beta$-CD can be fixed at $5 / 1$ for the fabrication of CDPU in further adsorption experiments. On the other hand, to facilitate regeneration, a solvent should be used that lowers the affinity, with pure ethyl acetate being a proper candidate.

3.4.1 Single-component adsorption kinetics. To investigate the adsorption processes of CAc and CA onto CDPU, kinetics of adsorption was conducted at $25{ }^{\circ} \mathrm{C}$. The changes in the amount of the guest sorption as a function of time are shown in Fig. 6 a. The guests exhibit rapid uptake within the first 50 min due to the existence of many available adsorption sites on CDPU surface. With the reduction of available adsorption sites on the surface, CAc or CA molecules have to pass through the surface and get into the narrow channels of CDPU, where they encounter much greater resistance, thus leading to a slow adsorption progress. The adsorption reaches saturation within about $3 \mathrm{~h}$. In addition, the adsorption amount of CAc is always much higher than that of CA during the adsorption process. 
(a)

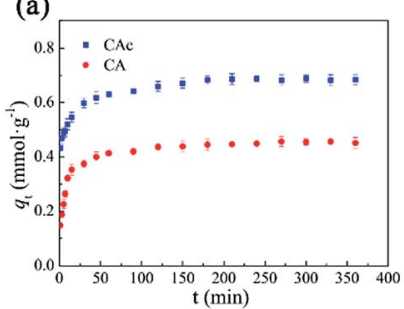

(b)

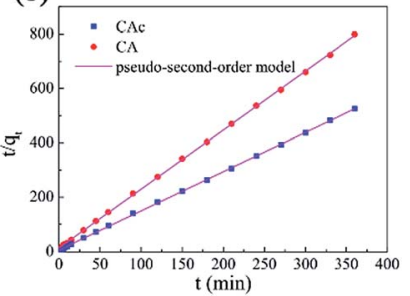

Fig. 6 The adsorptive kinetic curves in single-component system (a) and fitted by the pseudo-second-order model (b).

This agrees well with the theoretical observation that the adsorption sites of CDPU interact with CAc stronger than CA.

Three kinetic models, that are, the pseudo-first-order, pseudo-second-order and intra-particle diffusion models as shown in eqn (5)-(7), ${ }^{37}$ were employed to explore the adsorption kinetic behavior.

$$
\begin{gathered}
q_{t}=q_{\mathrm{e}}\left(1-\mathrm{e}^{-k_{1} t}\right) \\
q_{t}=k_{2} q_{\mathrm{e}}^{2} t /\left(1+k_{2} q_{\mathrm{e}} t\right) \\
q_{t}=k_{\mathrm{p}} t^{1 / 2}+C
\end{gathered}
$$

where $q_{t}$ and $q_{\mathrm{e}}\left(\mathrm{mmol} \mathrm{g}^{-1}\right)$ are the amount of CAc or CA adsorbed at time $t$ and equilibrium time; $k_{1}\left(\min ^{-1}\right), k_{2}(\mathrm{~g}$ $\left.\mathrm{mmol}^{-1} \mathrm{~min}^{-1}\right)$ and $k_{\mathrm{p}}\left(\mathrm{g} \mathrm{mmol}^{-1} \mathrm{~min}^{-1 / 2}\right)$ are rate constants of the pseudo-first-order, the pseudo-second-order and the intraparticle diffusion models, respectively; $C\left(\mathrm{mmol} \mathrm{g}^{-1}\right)$ is the affinity coefficient related to the boundary layer thickness.

The adsorption kinetic parameters, calculated from the three kinetic models above, are given in Table 1. For both CAc and CA, the values of the regression coefficients obtained from the pseudo-second-order model $(>0.99)$ are greater than that of the pseudo-first-order model, indicating that the adsorption kinetics can be more accurately represented by the pseudo-second-order model (Fig. 6b). In addition, for the intra-particle diffusion model, the regression coefficient $R^{2}$ is larger than 0.95 during the initial stage of the adsorption, revealing that the intra-particle diffusion process is the rate-limiting step of the adsorption in our study.

3.4.2 Single-component adsorption isotherms. The adsorption isotherms of CAc and CA onto CDPU in singlecomponent systems at 25,35 and $45{ }^{\circ} \mathrm{C}$ are plotted in Fig. 7. The equilibrium sorption amounts of CAc and CA increase with the initial concentrations of CAc and CA increasing, thus suggesting that a high initial concentration provides a significant

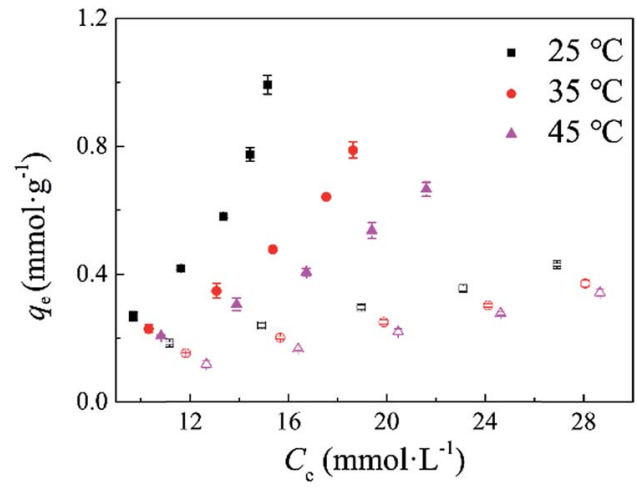

Fig. 7 Adsorption isotherms in single-component systems at 25, 35 and $45^{\circ} \mathrm{C}$ (solid symbols: CAc, open symbols: CA).

driving force during adsorption between the aqueous and solid phases. Meanwhile, the increase of the adsorption amount was observed with decreasing adsorption temperature. At $25{ }^{\circ} \mathrm{C}$, the equilibrium amount of CAc $\left(0.99 \mathrm{mmol} \mathrm{g}^{-1}\right)$ is larger apparently than that of CA $\left(0.43 \mathrm{mmol} \mathrm{g}^{-1}\right)$. This is probably because of the stronger interaction of CAc molecules with the adsorption sites of CDPU by hydrogen-bonding, hydrophobic and $\pi-\pi$ interactions. The sorption profiles of CAc and CA onto CDPU could be adequately reproduced by the Freundlich isotherm model (eqn (8)): ${ }^{38}$

$$
q_{\mathrm{e}}=K_{F} C_{\mathrm{e}}^{1 / n}
$$

where $q_{\mathrm{e}}$ and $C_{\mathrm{e}}$ are equilibrium adsorption capacity $\left(\mathrm{mmol} \mathrm{g}^{-1}\right)$ and the equilibrium concentration of CAc or CA $\left(\mathrm{mmol} \mathrm{L}^{-1}\right)$, $K_{\mathrm{F}}\left(\mathrm{mmol} \mathrm{g}^{-1}\left(\mathrm{mmol} \mathrm{L}^{-1}\right)^{-1 / n}\right)$ and $n$ are isotherm constants, obtained from the slopes and intercepts.

The parameters calculated from Freundlich isotherm at different temperature are listed in Table 2. The experimental data were well fitted by the Freundlich isotherm, indicating the adsorption of CAc and CA onto CDPU is a multilayer adsorption behavior.

3.4.3 Adsorption thermodynamics. The dependence of adsorption isotherms on temperature can be used to predict thermodynamics parameters, including Gibbs free energy change $\Delta G^{0}\left(\mathrm{~kJ} \mathrm{~mol}{ }^{-1}\right)$, enthalpy change $\Delta H^{0}\left(\mathrm{~kJ} \mathrm{~mol}^{-1}\right)$ and entropy change $\Delta S^{0}\left(\mathrm{~J} \mathrm{~mol}^{-1} \mathrm{~K}^{-1}\right)$, which were calculated according to eqn (9) and (10). ${ }^{39}$

$$
\begin{gathered}
\Delta G^{0}=-R T \ln K \\
\Delta G^{0}=\Delta H^{0}-T \Delta S^{0}
\end{gathered}
$$

\begin{tabular}{|c|c|c|c|c|c|c|c|c|c|}
\hline \multirow[b]{2}{*}{ Guest } & \multirow[b]{2}{*}{$q_{\mathrm{e}}(\exp )\left(\mathrm{mmol} \mathrm{g}^{-1}\right)$} & \multicolumn{3}{|c|}{ Pseudo-first-order model } & \multicolumn{3}{|c|}{ Pseudo-second-order model } & \multicolumn{2}{|c|}{$\begin{array}{l}\text { Intra-particle diffusion } \\
\text { model }\end{array}$} \\
\hline & & $k_{1}\left(\min ^{-1}\right)$ & $q_{\mathrm{e}}\left(\mathrm{mmol} \mathrm{g}^{-1}\right)$ & $R_{1}^{2}$ & $k_{2}\left(\mathrm{~g} \mathrm{mmol}^{-1} \mathrm{~min}^{-1}\right)$ & $q_{\mathrm{e}}\left(\mathrm{mmol} \mathrm{g}^{-1}\right)$ & $R_{2}{ }^{2}$ & $k_{\mathrm{p}}\left(\mathrm{g} \mathrm{mg}^{-1} \min ^{-1 / 2}\right)$ & $R_{\mathrm{P}}^{2}$ \\
\hline CAc & 0.69 & 1.07 & 0.64 & 0.55 & 0.40 & 0.69 & 0.99 & 0.04 & 0.98 \\
\hline $\mathrm{CA}$ & 0.46 & 0.18 & 0.43 & 0.93 & 0.38 & 0.46 & 0.99 & 0.08 & 0.97 \\
\hline
\end{tabular}

Table 1 Kinetic parameters for the adsorption of CAC and CA on CDPU at $25^{\circ} \mathrm{C}$ 
Table 2 Freundlich model parameters for single-component adsorption of CAC and CA on CDPU

\begin{tabular}{|c|c|c|c|c|c|c|}
\hline Parameters & \multicolumn{3}{|l|}{ CAc } & \multicolumn{3}{|l|}{$\mathrm{CA}$} \\
\hline$K_{\mathrm{F}}$ & $17.5 \times 10^{-3}$ & $11.5 \times 10^{-3}$ & $4.5 \times 10^{-3}$ & $0.65 \times 10^{-3}$ & $1.3 \times 10^{-3}$ & $4.2 \times 10^{-3}$ \\
\hline$n$ & 1.03 & 0.96 & 0.77 & 0.38 & 0.46 & 0.61 \\
\hline
\end{tabular}

Table 3 Thermodynamic parameters for adsorption of CAc and CA on CDPU

\begin{tabular}{|c|c|c|c|c|c|c|c|c|}
\hline Guests & \multicolumn{3}{|l|}{$K$} & \multicolumn{3}{|c|}{$\Delta G^{0}\left(\mathrm{~kJ} \mathrm{~mol}^{-1}\right)$} & $\Delta H^{0}\left(\mathrm{~kJ} \mathrm{~mol}^{-1}\right)$ & $\Delta S^{0}\left(\mathrm{~J} \mathrm{~mol}^{-1} \mathrm{~K}^{-1}\right)$ \\
\hline CAc & 19.20 & 16.06 & 12.18 & -7.32 & -7.11 & -6.61 & -17.95 & -35.50 \\
\hline
\end{tabular}

where $T$ is the absolute sorption temperature $(\mathrm{K}), R$ is gas constant $\left(\mathrm{J} \mathrm{mol}{ }^{-1} \mathrm{~K}^{-1}\right)$, and $K$ is the equilibrium constant $\left(\mathrm{m}^{3} \mathrm{~mol}^{-1}\right)$. The value of $\ln K$ at a certain temperature was determined by plotting $\ln \left(q_{\mathrm{e}} / C_{\mathrm{e}}\right)$ versus $q_{\mathrm{e}}$ and extrapolating $q_{\mathrm{e}}$ to zero.

The obtained thermodynamic parameters are listed in Table 3. The negative values of $\Delta G^{0}$ and negative values of $\Delta H^{0}$ confirm the thermodynamically feasible and exothermic nature of the adsorption of CAc and CA onto CDPU, and the more negative values of $\Delta G^{0}$ for CAc adsorbed onto CDPU than did CA indicates that CAc is more favorably adsorbed onto CDPU than CA. In addition, the negative values of $\Delta S^{0}$ show the decrease in randomness at the solid-solution interface during sorption. Normally, $\Delta H^{0}$ between -40 and $-120 \mathrm{~kJ} \mathrm{~mol}^{-1}$ are attributed to chemisorption, otherwise it indicates physisorption. ${ }^{34}$ Therefore, according to the $\Delta H^{0}$ values (CA: $-31.23 \mathrm{~kJ} \mathrm{~mol}^{-1}$,
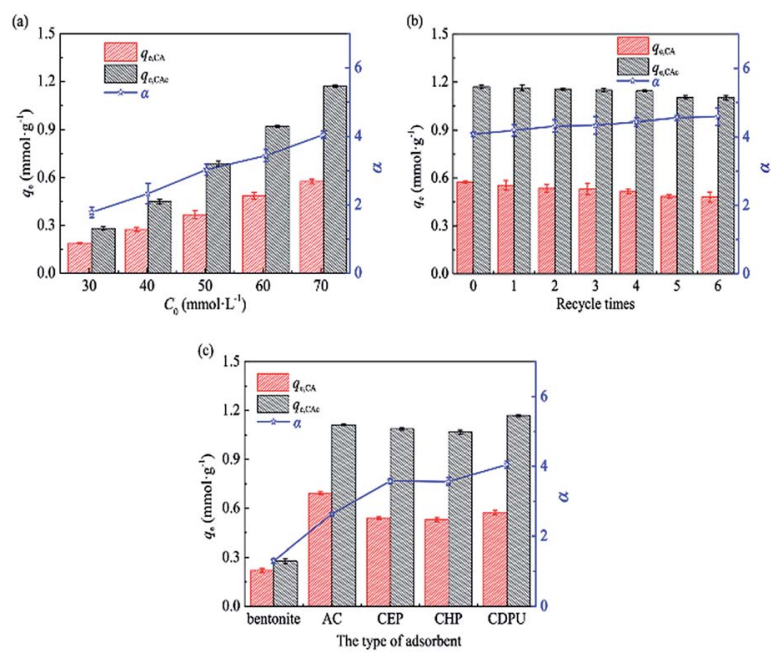

Fig. 8 Equimolar binary-component adsorption: (a) at different

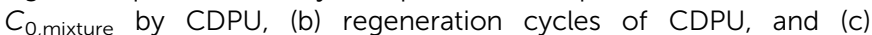
comparison of adsorption performances between CDPU and other adsorbents.
CAc: $-17.95 \mathrm{~kJ} \mathrm{~mol}^{-1}$ ), it can be confirmed that uptake of CAc and CA onto CDPU is a physisorption process, contributed by the combination of hydrogen bonding, hydrophobic and $\pi-\pi$ interactions, etc.

3.4.4 Binary-component equilibrium adsorption. To confirm the selective adsorption of CAc over CA under mixture conditions, we performed two-component adsorption experiments as a representative case. The adsorptive results of the equimolar mixture of CAc and CA in Fig. 8a show that the adsorption amounts of CAc and CA both increase with increasing the initial concentrations of guests. The maximum experimental uptakes of CAc and CA were 1.18 and $0.57 \mathrm{mmol} \mathrm{g}^{-1}$ at the highest initial concentration tested, which are obviously higher than that in single-component isotherm, indicating that the cooperative effect occurs in the adsorption of $\mathrm{CA} / \mathrm{CAc}$ mixtures onto CDPU. To interpret this finding we assume that the lateral Lewis acid-base interaction is formed between guest molecules as the lone-pair electrons on oxygen atom in guest molecules (acting as electron donor) and the respective empty molecular orbital of carbon atom in carbonyl group (acting as electron acceptor) can attract with each other through formation of an electron donor-acceptor complex. ${ }^{40}$ In addition, there is aromatic $\pi-\pi$ stacking interaction between the $\pi$-electron-deficient phenyl ring group in CA and the $\pi$-electronrich phenyl ring units in CAc, similar to the observation reported by J. Callison. ${ }^{\mathbf{4 1}}$ Interestingly, such cooperative adsorption can also permit improved selectivity of CDPU to CAc over CA, revealed by the increased CAc/CA selectivity with increasing the initial concentrations of guests. The highest selectivity (ca. 4.0) was obtained at $C_{0}=70 \mathrm{mmol} \mathrm{L}^{-1}$ (i.e., initial concentration of each component was $35 \mathrm{mmol} \mathrm{L}^{-1}$ ). As the initial concentrations of mixture increases, the uptake of CAc is more enhanced by the cooperative effect than that of CA. This results in a significant increase of the CAc/CA selectivity with increasing concentration, a characteristic that is favorable for application of CDPU to industrial adsorptive separation of CAc and CA. 
3.4.5 Recycle adsorption in binary solute mode. Further recycle experiments were conducted after the guest-saturated adsorbent was regenerated simply by desorption with ethyl acetate, a very mild protocol for generation. As shown in Fig. 8b, after six successive adsorption-desorption cycles, the adsorption capacity of CDPU decrease slightly from 1.18 to $1.10 \mathrm{mmol} \mathrm{g}^{-1}$ for CAc and from 0.57 to $0.48 \mathrm{mmol} \mathrm{g}^{-1}$ for CA, respectively. More importantly, the selectivity is almost unchanged after six circles. Therefore, CDPU has potential in industrial application due to its long-term stability and continuous high level of separation efficiency.

3.4.6 Comparison of adsorption properties with other sorbents. The adsorption capacity of CDPU and its selectivity for CAc over CA was compared with a number of sorbents, including the commercial bentonite and AC, two $\beta$-CD polymers crosslinked with aliphatic crosslinking agents (EPI and HDI). As shown in Fig. 8c, only a small portion of guests is adsorbed onto bentonite, and no adsorption selectivity of CAc is observed. For comparison, AC outperforms bentonite obviously, however, its selectivity is inferior than that of $\beta$-CD polymers. In addition, it is verified that CDPU exhibits the highest adsorption capacity and selectivity for CAc among the $\beta$-CD polymers, which may be attributed to $\pi-\pi$ interaction between the aromatic skeleton in the structure of CDPU and the guests.

\subsection{Mechanism analysis}

Based on the results of characterization, theoretical calculation and adsorption experiments, the possible adsorption mechanism of CA/CAc onto CDPU is given in Scheme 1. On the one hand, the adsorption of CA/CAc onto CDPU probably originates from non-covalent interaction (e.g., hydrogen bonding) according to the results of XPS and FTIR of after adsorption. On the other hand, the binding energies of CAc onto CDPU are higher than that of CA onto CDPU, which may be due to the stronger multiple noncovalent interactions between CDPU and CAc than that between CDPU and CA. Experimentally, we have observed clearly that CDPU displayed selective adsorption of CAc over CA, and moreover, the results of adsorption experiments showed that adsorption amount was enhanced in the presence of the co-solute, which was attributed to the

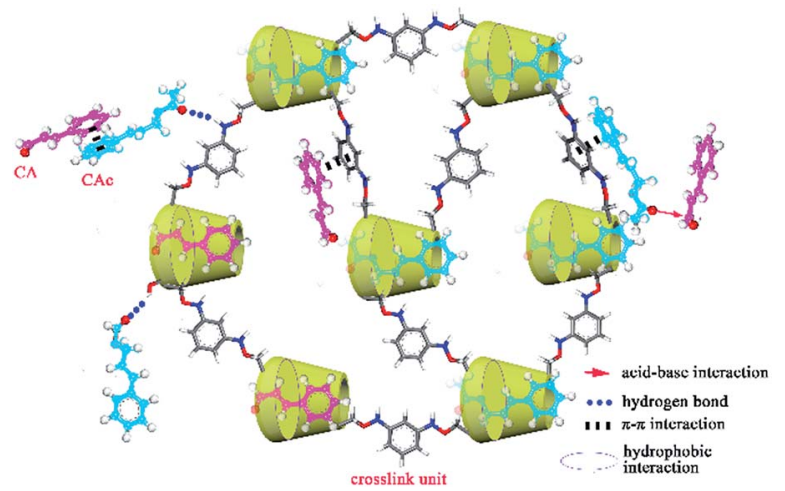

Scheme 1 The proposed adsorption mechanism of CAC and CA onto CDPU. cooperative effect arising from the lateral Lewis acid-base and $\pi-\pi$ interactions between CAc and CA molecules on the adsorbent surface. Therefore, based on the analysis above, hydrogen bonding interaction and other non-covalent interactions allow a preferential adsorption of CAc over CA. In addition, cooperative effect is found in CAc or CA adsorbed onto CDPU, due to Lewis acid-base and $\pi-\pi$ stacking between the guests themselves on the CDPU surface.

\section{Conclusion}

For adsorptive separation of CAc and CA, a polymer adsorbent of $\beta$-cyclodextrin polyurethane (CDPU) was synthesized by crosslinking $\beta$-CD with TDI. The results of instrumental analyses and DFT calculations demonstrated that CAc showed superior affinity to CDPU than CA, due to the stronger noncovalent interactions between CAc and CDPU than that between CA and CDPU. Therefore, CAc could be adsorbed onto CDPU more effectively than CA, indicating a selective adsorption property of CDPU. In single-component adsorption systems, the adsorption of CAc or CA onto CDPU followed the pseudo-second-order kinetics and Freundlich adsorption isotherm models. And the thermodynamics study indicated spontaneous and exothermic nature of CAc or CA uptake onto CDPU. Moreover, the uptakes of CAc and CA in binary equimolar components adsorption systems were apparently higher than the corresponding ones in single-component adsorption systems, presumably resulting from the cooperative effect caused by Lewis acid-base and $\pi-\pi$ stacking interactions between the adsorbed CAc and CA molecules. Furthermore, the greater uptake enhancement and cooperative effect of CAc in the binary-component adsorption systems are probably due to its stronger adsorption affinity to CDPU than CA. In binarycomponent systems, the maximum uptakes were 1.18 and $0.57 \mathrm{mmol} \mathrm{g}^{-1}$ for CAc and CA at $C_{0}=70 \mathrm{mmol} \mathrm{L}^{-1}$, giving the highest selectivity of $c a$. 4.0. Based on the theoretical analyses and adsorptive results, the mechanism for selective adsorption onto CDPU is assumed to be the combination of multiple weak interactions, including hydrogen bonding, hydrophobic, and $\pi-\pi$ interactions. The results of this study indicate that the CDPU exhibits an excellent adsorption capacity and high separation efficiency even after six adsorption/desorption cycles, making this material promising for adsorptive CAc/CA separation processes.

\section{Conflicts of interest}

There are no conflicts to declare.

\section{Acknowledgements}

This work was supported by the Natural Science Foundation of Guangxi Province (No. 2015GXNSFBA139030, 2015GXNSFDA139005), the National Natural Science Foundation of China (21606054). The special support for the application of computing science from High-performance Computing Center of Guangxi University was also acknowledged. 


\section{Notes and references}

1 Y. H. Wang, B. Avula, N. P. D. Nanayakkara, J. P. Zhao and

I. A. Khan, J. Agric. Food Chem., 2013, 61, 4470-4476.

2 P. Goni, P. Lopez, C. Sanchez, R. Gomez-Lus, R. Becerril and C. Nerin, Food Chem., 2009, 116, 982-989.

3 T. A. F. Ferro, J. M. M. Araujo, B. L. D. Pinto, J. S. dos Santos, E. B. Souza, B. L. R. da Silva, V. L. P. Colares, T. M. G. Novais, C. M. B. Filho, C. Struve, J. B. Calixto, V. Monteiro-Neto, L. C. N. da Silva and E. S. Fernandes, Front Microbiol., 2016, 7, 1-10.

4 Y. Zhang, Y. Fang, Z. Yang and H. Ji, Fine Chem., 2014, 31, 5053.

5 S.-C. Lee, W.-X. Xu, L.-Y. Lin, J.-J. Yang and C.-T. Liu, J. Agric. Food Chem., 2013, 61, 4905-4913.

6 R. Ribeiro-Santos, M. Andrade, D. Madella, A. P. Martinazzo, L. de Aquino Garcia Moura, N. R. de Melo and A. SanchesSilva, Trends Food Sci. Technol., 2017, 62, 154-169.

7 Y.-T. Tung, M.-T. Chua, S.-Y. Wang and S.-T. Chang, Bioresour. Technol., 2008, 99, 3908-3913.

8 J. Cocchiara, C. S. Letizia, J. Lalko, A. Lapczynski and A. M. Api, Food Chem. Toxicol., 2005, 43, 867-923.

9 Z. Yang and H. Ji, ACS Sustainable Chem. Eng., 2013, 1, 11721179.

10 P. D. Tomke and V. K. Rathod, Ultrason. Sonochem., 2015, 27, 241-246.

11 A. Wolfson, C. Dlugy, A. Karanet and D. Tavor, Tetrahedron Lett., 2012, 53, 4565-4567.

12 K. Chai, Y. Zhang, Z. Yang, Y. Xuang and H. Ji, Chem. Ind. Eng. Pro., 2014, 33, 475.

13 R. Wang, B. Yu, X. S. Jiang and J. Yin, Adv. Funct. Mater., 2012, 22, 2606-2616.

14 L. F. Wang, B. D. Sun, F. H. Yang and R. T. Yang, Chem. Eng. Sci., 2012, 73, 208-217.

15 Z. Luo, H. Shu, P. Guo, P. Zheng, X. Pan, W. Du, R. Liu, A. Zeng, C. Chang and Q. Fu, RSC Adv. , 2016, 6, 15549-15557.

16 R. F. DeJaco, P. Bai, M. Tsapatsis and J. I. Siepmann, Langmuir, 2016, 32, 2093-2101.

17 A. Alsbaiee, B. J. Smith, L. Xiao, Y. Ling, D. E. Helbling and W. R. Dichtel, Nature, 2016, 529, 190-194.

18 F. Zhao, E. Repo, D. Yin, Y. Meng, S. Jafari and M. Sillanpää, Environ. Sci. Technol., 2015, 49, 10570-10580.

19 H. Li, B. Meng, S.-H. Chai, H. Liu and S. Dai, Chem. Sci., 2016, 7, 905-909.

20 L. Xiao, Y. Ling, A. Alsbaiee, C. Li, D. E. Helbling and W. R. Dichtel, J. Am. Chem. Soc., 2017, 139, 7689-7692.
21 F. van de Manakker, T. Vermonden, C. F. van Nostrum and W. E. Hennink, Biomacromolecules, 2009, 10, 3157-3175.

22 B. Meng, H. Li, S. M. Mahurin, H. Liu and S. Dai, RSC Adv., 2016, 6, 110307-110311.

23 J. Zhao, T. T. Liu and G. Chen, React. Funct. Polym., 2016, 102, 119-129.

24 M. H. Mohamed, L. D. Wilson and J. V. Headley, Carbohydr. Polym., 2010, 80, 186-196.

25 M. Bhaskar, P. Aruna, R. J. Ganesh Jeevan and G. Radhakrishnan, Anal. Chim. Acta, 2004, 509, 39-45.

26 C. P. Okoli, G. O. Adewuyi, Q. Zhang and Q. Guo, RSC Adv., 2016, 6, 71596-71611.

27 T. Hishiya, H. Asanuma and M. Komiyama, J. Am. Chem. Soc., 2002, 124, 570-575.

28 V. Abbate, A. R. Bassindale, K. F. Brandstadt and P. G. Taylor, Polym. Chem., 2012, 3, 2018-2027.

29 H. Yamasaki, Y. Makihata and K. Fukunaga, J. Chem. Technol. Biotechnol., 2008, 83, 991-997.

30 M. Maes, L. Alaerts, F. Vermoortele, R. Ameloot, S. Couck, V. Finsy, J. F. M. Denayer and D. E. De Vos, J. Am. Chem. Soc., 2010, 132, 2284-2292.

31 X. Wang, M. Ding, Z. Liu and D. Wang, RSC Adv., 2015, 5, 19541-19551.

32 M. Raoov, S. Mohamad and M. R. Abas, J. Hazard. Mater., 2013, 263, 501-516.

33 M. H. Mohamed, L. D. Wilson, J. V. Headley and K. M. Peru, Phys. Chem. Chem. Phys., 2011, 13, 1112-1122.

34 K. Lu, K. Chai, Q. Liang, Z. Xu, G. Li and H. Ji, Chem. Eng. J., 2017, 317, 862-872.

35 T. Steiner, Angew. Chem., Int. Ed., 2002, 41, 48-76.

36 M. Maes, M. Trekels, M. Boulhout, S. Schouteden, F. Vermoortele, L. Alaerts, D. Heurtaux, Y.-K. Seo, Y. K. Hwang, J.-S. Chang, I. Beurroies, R. Denoyel, K. Temst, A. Vantomme, P. Horcajada, C. Serre and D. E. De Vos, Angew. Chem., 2011, 123, 4296-4300.

37 M. M. Galangash, Z. N. Kolkasaraei, A. Ghavidast and M. Shirzad-Siboni, RSC Adv., 2016, 6, 113492-113502.

38 J.-M. Lv, Y.-L. Ma, X. Chang and S.-B. Fan, Chem. Eng. J., 2015, 273, 247-253.

39 F. Zhu, Y. Lu and L. Li, RSC Adv., 2016, 6, 111120-111128.

40 W. Zhang, Z. W. Xu, B. C. Pan, Q. J. Zhang, Q. R. Zhang, W. Du, B. J. Pan and Q. X. Zhang, Sep. Purif. Technol., 2007, 55, 141-146.

41 J. Callison, F. Betzler, K. de Cuba, W. van der Borden, K. van der Velde, R. H. Carr, H. M. Senn, L. J. Farrugia, J. M. Winfield and D. Lennon, Ind. Eng. Chem. Res., 2012, 51, 11021-11030. 